\title{
Original
}

Feser, F.; Storch, H.v.:

A Dynamical Downscaling Case Study for Typhoons in Southeast Asia Using a Regional Climate Model

In: Monthly Weather Review (2008) AMS

DOI: 10.1175/2007MWR2207.1 


\title{
NOTES AND CORRESPONDENCE
}

\section{A Dynamical Downscaling Case Study for Typhoons in Southeast Asia Using a Regional Climate Model}

\author{
Frauke Feser and Hans von STORCH \\ Institute for Coastal Research, GKSS Research Centre, Geesthacht, Germany
}

(Manuscript received 27 March 2007, in final form 13 August 2007)

\begin{abstract}
This study explores the possibility of reconstructing the weather of Southeast Asia for the last decades using an atmospheric regional climate model, the Climate version of the Lokal-Modell (CLM). For this purpose global National Centers for Environmental Prediction-National Center for Atmospheric Research (NCEP-NCAR) reanalyses data were dynamically downscaled to $50 \mathrm{~km}$ and in a double-nesting approach to $18-\mathrm{km}$ grid distance. To prevent the regional model from deviating significantly from the reanalyses with respect to large-scale circulation and large-scale weather phenomena, a spectral nudging technique was used.

The performance of this technique in dealing with Southeast Asian typhoons is now examined by considering an ensemble of one simulated typhoon case. This analysis is new insofar as it deals with simulations done in the climate mode (so that any skill of reproducing the typhoon is not related to details of initial conditions), is done in ensemble mode (the same development is described by several simulations), and is done with a spectral nudging constraint (so that the observed large-scale state is enforced in the model domain). This case indicates that tropical storms that are coarsely described by the reanalyses are correctly identified and tracked; considerably deeper core pressure and higher wind speeds are simulated compared to the driving reanalyses. When the regional atmospheric model is run without spectral nudging, significant intraensemble variability occurs; also additional, nonobserved typhoons form. Thus, the insufficiency of lateral boundary conditions alone for determining the details of the dynamic developments in the interior becomes very clear. The same lateral boundary conditions are consistent with different developments in the interior. Several sensitivity experiments were performed concerning varied grid distances, different initial starting dates of the simulations, and changed spectral nudging parameters.
\end{abstract}

\section{Introduction}

For assessing risks related to weather phenomena as well as for identification of anthropogenic signals in the weather record, long homogeneous time series are needed. In the case of marine phenomena, the interest is mostly with wind statistics, in particular strong wind incidents, that is, wind storms. Unfortunately, long homogeneous time series of wind speeds are rarely available because of the sensitivity of recording wind to local environmental change, changing instrumentation, and observation practice (Bärring and von Storch 2004).

Corresponding author address: Frauke Feser, Institute for Coastal Research, GKSS Research Centre, 21502 Geesthacht, Germany.

E-mail: feser@gkss.de
In recent years, a dynamical downscaling strategy has been developed. It has been applied to the problem of determining a high-resolution hindcast of the weather of western Europe for the last decades (Feser et al. 2001; Sotillo et al. 2005; Weisse et al. 2005), and of seasons in Southeast Asia (Lee et al. 2004; Kang et al. 2005). The idea is to force large-scale synoptic information provided by reanalyses, such as those prepared by the National Centers for Environmental PredictionNational Center for Atmospheric Research (NCEPNCAR; Kalnay et al. 1996), upon a regional atmospheric model (RCM; von Storch et al. 2000; MiguezMacho et al. 2004). A similar approach has also been used for oceanographic simulations (see Wright et al. 2006).

The main goal in downscaling is to obtain regional weather phenomena that are influenced by the local 
orography, land-sea contrast, and small-scale atmospheric features (e.g., convection). The dynamical downscaling is supposed to retain all the large-scale information that can be resolved well by the global model or reanalyses. It should also add regional information that the coarse-resolution global model could not generate. For this purpose limited-area models require meteorological information at their boundaries from either a global climate model or reanalysis data. But, regional models have to deal with the mathematically not-well-posed problem of lateral boundary conditions. That is, there is in general no well-defined solution fitting all prescribed boundary conditions in the model interior. There may be several or even no solutions fitting both the model equations and the boundary conditions; therefore, in general no unique solution can be specified as demanded in mathematical terms. This effect will be demonstrated later in this article. The inconsistencies between the model solution and the global forcing field along the boundaries produce noise and often instabilities. This problem was efficiently rectified by the inclusion of a sponge zone (Davies 1976). This broadened buffer zone features exponentially decreasing weights ensuring the consistency of the interior flow with the boundary conditions at the outflow boundaries.

Castro et al. (2005) examined to what degree boundary conditions steer processes in the limited-area model interior. They found that the larger model domains become, the less influential the lateral boundary conditions become for the variability of small-scale features, while at the same time surface boundaries exert relatively greater control on the RCM solution. Their results also showed that the influence of the surface boundary forcing was highly dependent on the model experimental design, such as domain size, nudging options, specification of the surface boundary itself, and model parameterization schemes.

If the regional model is driven only by boundary conditions without controlling large-scale weather phenomena in the model interior some problems with large-scale features may occur, especially for blocking weather situations. Thereby the domain size is an important factor. Large model areas are more affected by large-scale deviations between the global and regional model. But also the exchange rate between the lateral boundaries and the inner model domain has an immediate effect on the large-scale constraint the driving global model exerts on the limited-area model. Rinke and Dethloff (2000) showed that in the circumpolar Arctic domain the lateral boundary control is weaker compared to that in model areas of similar domain size in midlatitude areas, which are regularly "flushed" by the normally eastward circulation through the area. They also showed that RCMs may develop systematic errors in the simulation of the large-scale flow if the boundary forcing is weak.

Global and regional climate models have their individual best simulation performance at different spatial scales. Global models perform best for large spatial scales, a distance of five to six grid boxes, and more are often considered to be trustworthy. This lies in the range of about $800 \mathrm{~km}$ and larger, depending on the global model's resolution. In the downscaling approach (von Storch 1995), the regional model should not modify these scales well resolved by the global model. Laprise (2003) introduced a technique to identify the resolved scales and the nonlinear interactions between these scales in nested limited-area models by adopting a spectral perspective.

Kida et al. (1991) presented a new concept of a spectral boundary to join global and regional climate models. In this technique large-scale fields of a limited-area model are replaced with the corresponding large-scale fields supplied externally from the global climate model at a selected regular time interval. It was then further developed by Waldron et al. (1996) to a method that allows imposition of the large-scale fields throughout the limited-area model domain. Von Storch et al. (2000) used and further refined the spectral nudging technique to nudge the long waves in the regional domain to those of the driving global reanalyses in a multidecadal climate hindcast study. This method adds nudging terms to the regional model solution that nudge the atmospheric fields toward the direction of the global driving fields. Horizontal wind components were nudged to match the driving reanalyses, but only at large spatial scales. Thereby the nudging coefficients were applied exclusively for low wavenumbers and above $850 \mathrm{hPa}$, with increasing strength for higher model levels. The method was also implemented into an RCM to study the East Asian summer monsoon (Kang et al. 2005). They found that the nudging led to improved model performance not only in simulating the mean features but also in capturing individual precipitation events. Recently, a new approach to nudge the large scales of the driving global model to a regional spectrally represented model was presented by Kanamaru and Kanamitsu (2007). In contrast to a gridpoint model, a spectral model only computes perturbations of a mean state given by the global forcing model. In this respect, this concept is strongly related to the idea of spectral nudging.

The issue of the added value provided by RCMs is still contested. A dedicated analysis of the output of a spectrally nudged RCM on different spatial scales has 
shown that the RCM performs better than the global reanalysis on medium scales $(250-550 \mathrm{~km})$, which are insufficiently resolved by the reanalysis (Feser 2006). For large spatial scales $(>700 \mathrm{~km})$ an added value was detected for heterogeneous variables like near-surface temperature, whereas the more homogeneous sea level pressure was not better described than by the driving NCEP reanalyses. With spectral nudging a slight improvement was found for subsynoptic scales, but without large-scale constraint the simulation got worse on all spatial scales. J. Winterfeldt (2007, personal communication) examined northeast Atlantic storms in the RCM dataset. On a case-by-case basis, not all storms are reproduced well, but if statistics are considered, the reproduction is satisfactory without bias and with a variability similar to the observed for coastal regions. Castro et al. (2005) analyzed the value restored and added by dynamical downscaling and found an added value especially for surface boundary forcings like variations in topography. If no interior nudging was used, the RCM had greater errors at large spatial scales as both horizontal grid spacing and domain size increased. They recommend usage of a spectral nudging technique to preserve the value added at the small scale while avoiding a large-scale kinetic energy loss.

A multidecadal hindcast for western Europe (Feser et al. 2001) showed that for certain weather conditions large deviations between the driving global reanalyses and the regional model may occur on large scales, if the simulation is not constrained on these scales. Significantly different simulation pattern emerged. This unfavorable feature was avoided by constraining the simulation with spectral nudging (von Storch et al. 2000).

Hourly high-resolution wind and air pressure fields of this regional hindcast have been used to assess changing ocean wave conditions (Soares et al. 2002; Gaslikova and Weisse 2006; Weisse and Plüss 2006), coastal currents, and storm surges (Weisse et al. 2005; Aspelien 2006) in the northeast Atlantic. The resulting datasets of ocean wave parameters and storm surge heights have been used in a number of impact studies related to offshore activities, ship construction, or coastal defense.

The authors suggest implementing a similar method to reconstruct tropical weather in Southeast Asia. Obviously, the key problem is the simulation of typhoons. Landman et al. (2005) have demonstrated that even with a relatively coarse resolution of $60 \mathrm{~km}$, tropical storm tracks and the vortex intensities are described by a regional atmospheric model (without large-scale constraint). Recently Camargo et al. (2007) explored the feasibility of downscaling seasonal tropical cyclone activity in a global model with observed sea surface tem- peratures using a regional model. Their results show that the representation of the tropical cyclones was improved but they were not as intense as observed cyclones. The regional model did not reproduce the lower number of tropical cyclones in 1998 (Nakazawa 2001) compared to 1994. Walsh et al. (2004) used dynamical downscaling to simulate tropical cyclones over Australia. They used a fine horizontal resolution of $30 \mathrm{~km}$, which resulted in a good climatological simulation of tropical cyclone formation. An experiment with enhanced greenhouse conditions showed an increase in tropical cyclone maximum intensities.

Obviously, the success of describing the climatology of tropical cyclones requires that the model is describing the large-scale circulation, in particular the monsoon system, adequately. Unconstrained regional climate model simulations often fail to do so. Fu et al. (2005) examined the quality of several RCMs for simulating the Asian monsoon climate within the regional climate model intercomparison project for Asia. Another study using reanalysis data as input for a regional model was presented by Chan et al. (2004). They modified the Regional Climate Model of the National Climate Center of China to simulate the summer monsoon rainfall over South China and the South China Sea more realistically. A 10-yr Southeast Asia regional climatology for May and June driven by reanalysis data was described by Liu et al. (2006). This model was able to reproduce the main features of the monsoon circulation and the vertical structure of the atmosphere as well as the intensification and northwestward movement of the upper anticyclone from May to June.

In the current study the performance of the dynamical downscaling method (using spectral nudging as a large-scale constraint) was explored in order to apply it to Southeast Asia. The results of constructing a very strong and large typhoon, namely, Winnie in August 1997, are described. The rationale of doing so is to determine how well individual storms are caught and described by this method, even if it is not expected that all details are described. At a later time, the performance of the approach in describing an ensemble of storms will be examined.

\section{Testing the approach with a typhoon: Winnie, August 1997}

Typhoon Winnie formed at $7^{\circ} \mathrm{N}, 167^{\circ} \mathrm{E}$ inside of the eastern part of the regional model domain (Fig. 1) on 6 August 1997. The RCM simulations are initialized with NCEP-NCAR reanalyses (in the following just called "NCEP" for brevity reasons) at or shortly after $1 \mathrm{Au}-$ gust 1997 . The storm itself is described by NCEP re- 


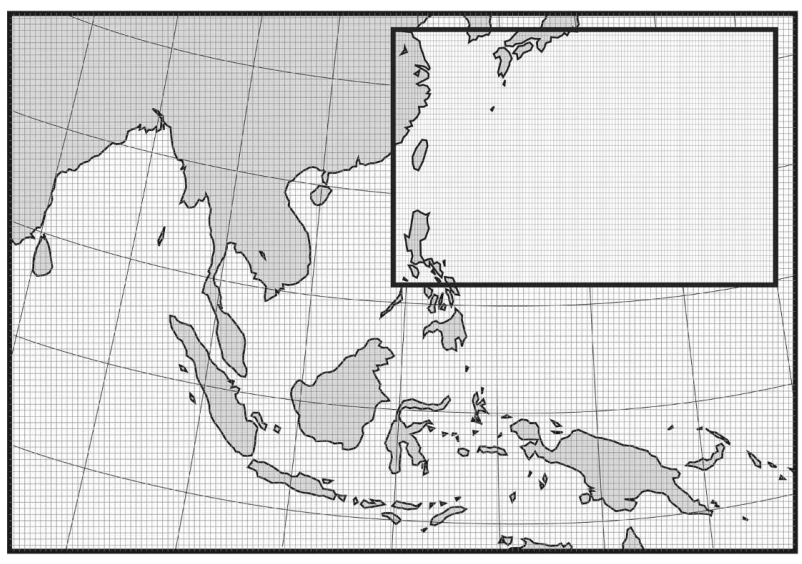

FIG. 1. Simulation area-the large area is described by a $50-\mathrm{km}$ grid, the smaller by an $18-\mathrm{km}$ grid. The grid boxes are only schematically depicted and do not represent the real gridbox size.

analyses with a minimum core pressure of $965 \mathrm{hPa}$ (at 0600 UTC) and maximum near-surface wind speeds of $29 \mathrm{~m} \mathrm{~s}^{-1}$ (at 1800 UTC) on 17 August 1997, which is too weak compared to the "best track data" [Japan Meteorological Agency (JMA), http://www.jma.go.jp/jma/ jma-eng/jma-center/rsmc-hp-pub-eg/trackarchives. html] of $915 \mathrm{hPa}$ and $51 \mathrm{~m} \mathrm{~s}^{-1}$ on 12 and 13 August 1997.

The state-of-the-art RCM CLM (http://www.clmcommunity.eu) is used, which is the climate version of the operational weather forecast model Lokal-Modell (LM; Steppeler et al. 2003) of the German Weather Service. Starting from the next model version 4.0, the LM and CLM will be merged and the user will be able to either select the climate or the forecast mode. The CLM is an RCM (which can be run in hydrostatic or nonhydrostatic mode) developed from the LM by the CLM community. Since 2005 it has been the community model of the German climate research. The model has been used for simulations on time scales up to centuries and spatial resolutions between 1 and $50 \mathrm{~km}$. The advantages of this RCM are mainly the possibility to use it in nonhydrostatic mode when increasing the horizontal resolution and that spectral nudging can be selected. It runs with standard parameterizations for physical processes; for convection the Kain-Fritsch parameterization scheme (Kain 2004) has been selected. The model was chosen to run with a $0.5^{\circ}$ grid, which corresponds to about $50 \mathrm{~km}$ (see Fig. 1). A sponge zone of eight grid points is used. The spectral nudging is applied to the two horizontal wind components: below $850 \mathrm{hPa}$ no nudging is used; beginning with $850 \mathrm{hPa}$ a weak nudging (corresponding to an $e$-folding decay time of an introduced disturbance of 20 days) is adopted, which increases with height to an $e$-folding

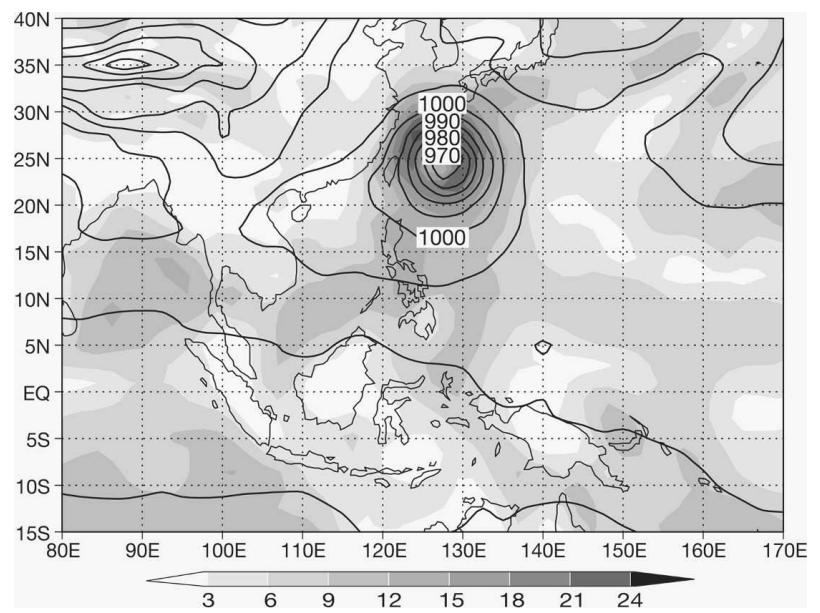

FIG. 2. Description of Winnie at 0000 UTC 17 Aug 1997 in the NCEP-NCAR reanalysis. The figure shows sea level pressure isobars $(\mathrm{hPa})$ and shaded near-surface wind speed fields $\left(\mathrm{m} \mathrm{s}^{-1}\right)$.

time of approximately $2 \mathrm{~h}$ at $100 \mathrm{hPa}$ (von Storch et al. 2000). The $e$-folding decay time describes the time that an introduced disturbance would need to be damped out by the model system. A stronger nudging coefficient will lead to shorter $e$-folding decay times. Nudged are all horizontal wind components on spatial scales larger than about $750 \mathrm{~km}$. Embedded into the $50-\mathrm{km}$ simulation region is a smaller region with a grid length of $18 \mathrm{~km}$ (Fig. 1). For this double nesting approach the boundary conditions are taken from the formerly computed regional simulation at a resolution of $50 \mathrm{~km}$. So actually two nested RCM simulations had to be computed, first from the global model to $50-\mathrm{km}$ resolution and then from the $50-\mathrm{km}$ regional run to $18 \mathrm{~km}$. Again a spectral nudging approach is employed; this time the constraint is taken from the regional $50-\mathrm{km}$ grid simulation. The vertical structure of the nudging is the same as before, but the constrained spatial scales are now reduced from the formerly used $750 \mathrm{~km}$ to $215 \mathrm{~km}$ and longer - this scale is considered to be simulated reliably by the $50-\mathrm{km}$ CLM.

The situation on 0000 UTC 17 August 1997, as described by the NCEP reanalysis, is shown in Fig. 2, with a core pressure of about $970 \mathrm{hPa}$ and $24 \mathrm{~m} \mathrm{~s}^{-1}$ maximum near-surface winds. In case of the spectrally nudged 50-km RCM simulation (Fig. 3) just one typhoon is also simulated, at the same location as in the NCEP reanalysis, but with a core pressure of about 935 $\mathrm{hPa}$ and maximum wind speeds of $36 \mathrm{~m} \mathrm{~s}^{-1}$.

The track of Typhoon Winnie is shown in Fig. 4 for the best-track data, NCEP reanalysis, and for four simulations with spectrally nudged CLM and four unconstrained CLM simulations. The NCEP track deviates only in the earlier part of the development from 


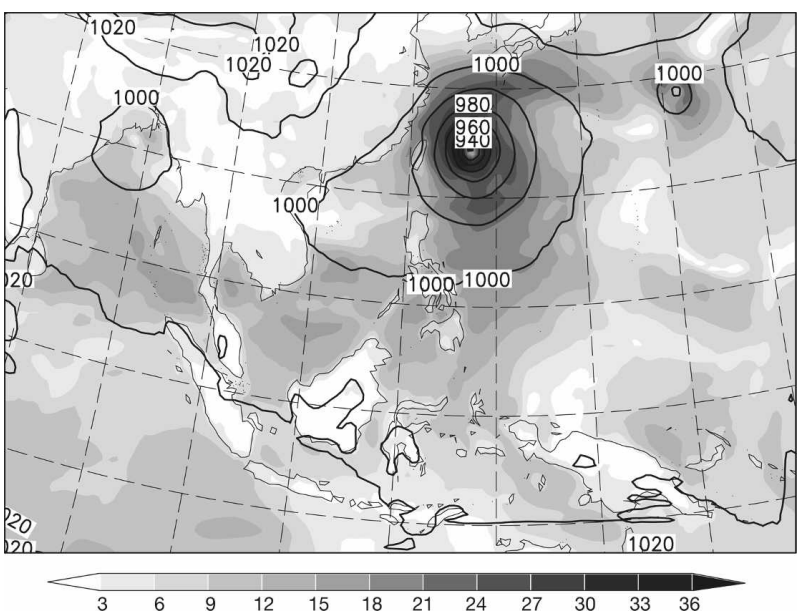

FIG. 3. Simulation of Winnie at 0000 UTC 17 Aug 1997 on the $50-\mathrm{km}$ grid with spectral nudging. The figure shows sea level pressure isobars $(\mathrm{hPa})$ and shaded near-surface wind speed fields $\left(\mathrm{m} \mathrm{s}^{-1}\right)$.

the best-track data; the spectrally nudged RCM simulations all follow the best-track data closely, even during the phase when NCEP deviates a bit. One simulation without spectral nudging is also close to the besttrack data, but the other unconstrained RCM simulations deviate to the north of the typhoon track. For the tracking a simple minimum pressure search was performed for the sea level pressure field. A comparison with more sophisticated methods by, for example, Hodges (1994, 1995) did not reveal any significant differences for this particular case study of Typhoon Winnie (not shown).
The temporal development of the typhoon, in terms of core pressure and maximum near-surface wind speed is shown in Fig. 5 for JMA best-track data, for NCEP reanalyses, and for different configurations of spectrally nudged CLM simulations. For the time being only the curve "CLM 0.5 degrees" is of relevance; the other curves refer to results obtained with higher resolution and are discussed later (see section 3). Obviously, the CLM simulation (gray solid line) creates significantly lower core pressure values and higher maximum nearsurface wind speeds than NCEP (gray dashed line). Minimum core pressure in NCEP is on 17 August about $965 \mathrm{hPa}$, whereas CLM generates about $930 \mathrm{hPa}$ at the same time. In the best-track data (gray dots), however, the maximum intensity is reached earlier, on 12 and 13 August, with a core pressure of $915 \mathrm{hPa}$. A similar situation holds for wind speed, which is about $29 \mathrm{~m} \mathrm{~s}^{-1}$ in NCEP and $40 \mathrm{~m} \mathrm{~s}^{-1}$ in CLM about 3.5 days after a maximum of more than $50 \mathrm{~m} \mathrm{~s}^{-1}$ in the best-track data.

To demonstrate the additional detail, two digital 2D isotropic filters [with a footprint of $2 \times 8+1$ grid points (Feser and von Storch 2005)] were applied. One filter approximately isolates "large" scales (diameters larger than about $625 \mathrm{~km}$ ) and another filter "medium" scales (diameters between 180 and $360 \mathrm{~km}$ ). Note that the filter response function only approximates an ideal response function with 1's for the retained scales and 0's for the suppressed scales. The actual response function deviates from the ideal form. The retained scales are somewhat dampened, and traces of the suppressed scales remain. The sum of the filtered components has less variability than the original field. The filter allows,

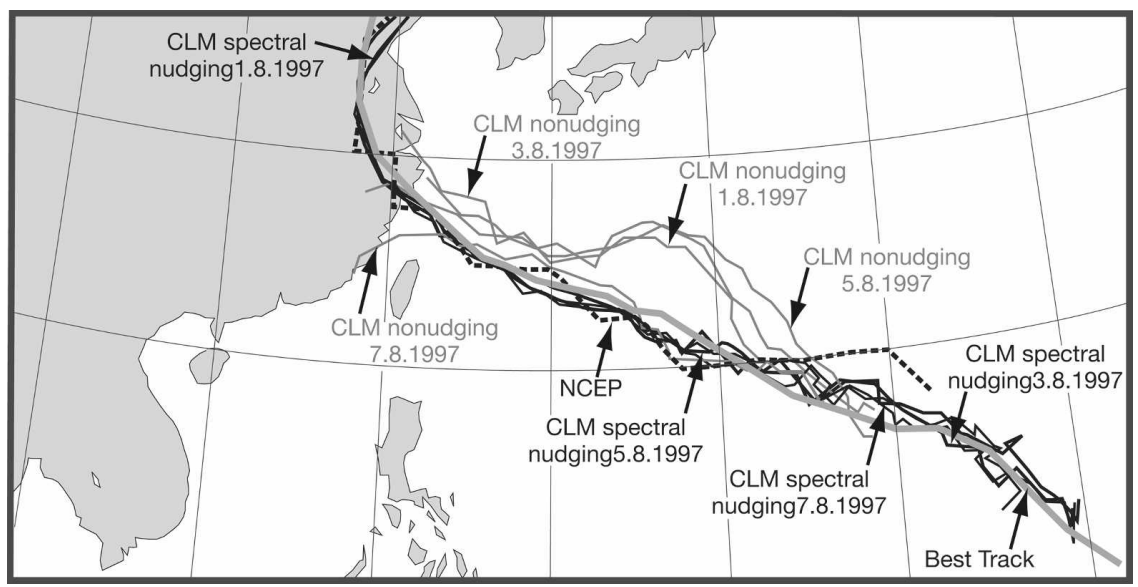

FIG. 4. Tracks of Winnie as given by the JMA best-track data (thick gray solid line), by NCEP-NCAR reanalyses (thick black dashed line), four simulations with CLM without spectral nudging (thin gray solid lines), and four with spectral nudging (thin black solid lines). The only difference between the individual CLM ensemble members with or without spectral nudging is their starting date at or shortly after 1 Aug 1997. 

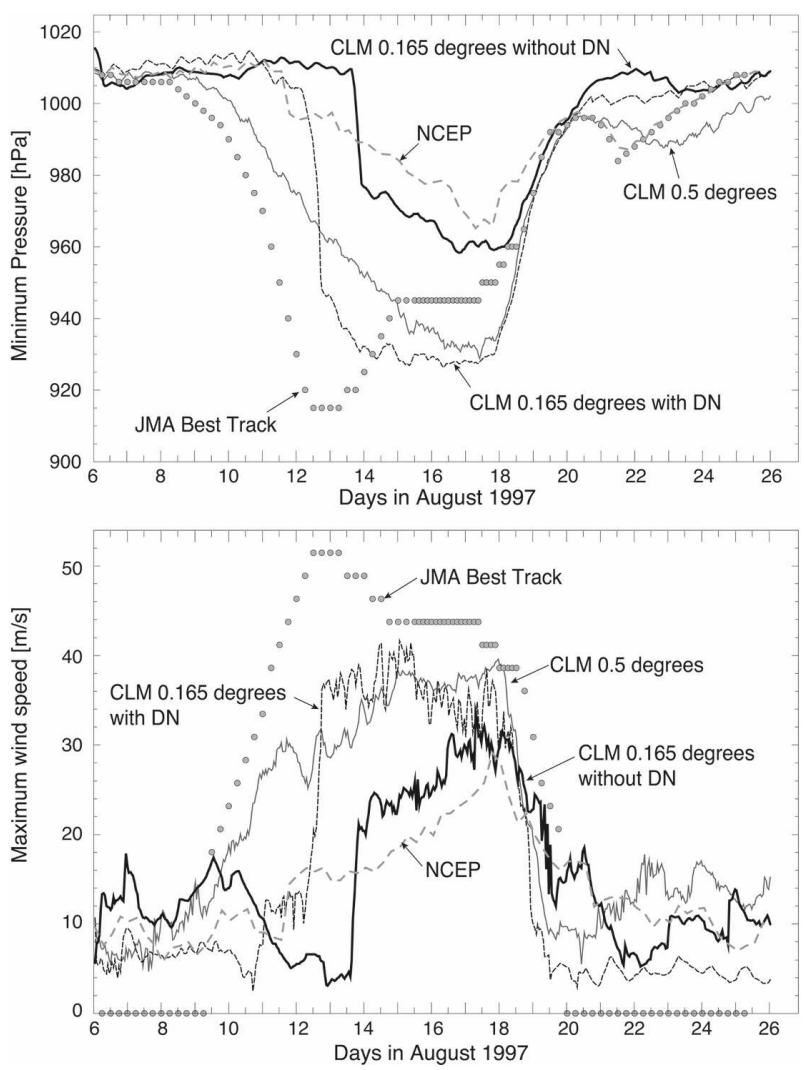

FIG. 5. Core pressure $(\mathrm{hPa})$ and maximum near-surface wind speed $\left(\mathrm{m} \mathrm{s}^{-1}\right)$ as given by the JMA best-track estimates, in the NCEP-NCAR reanalyses, in the CLM simulation with a $50-\mathrm{km}$ grid, and two CLM simulations with an $18-\mathrm{km}$ grid, either using the global NCEP reanalyses directly as input without double nesting ("without DN") or with double nesting using the grids shown in Fig. 1 ("with DN").

however, a comparison of scale contributions in different fields.

In Fig. 6 spatially filtered maps of air pressure for Taiwan and its surroundings are shown for NCEP (left column) and for the spectrally nudged RCM simulation (right column) initiated on 1 August 1997. Results are shown for NCEP and spectrally nudged CLM on 0000 UTC 17 August 1997. For that purpose, NCEP was interpolated to the $50-\mathrm{km}$ grid of CLM. To be able to distinguish between positive and negative values, negative values are plotted as dashed isolines.

The large-scale filtered component is dominated by Winnie's low pressure system with $20-\mathrm{hPa}$ deviation from mean pressure and less for NCEP and less than 25 hPa in CLM (Fig. 6). This circular low pressure system is modulated by medium-scale structures in both NCEP and CLM, with a further pressure deepening in the center ( $6 \mathrm{hPa}$ in NCEP; $8 \mathrm{hPa}$ in CLM), and an attenuation of the low pressure in a surrounding ring. Obviously, the regional climate model adds significant re- gional detail; that this is actually an improvement is indicated by the deepening of the core pressure (Fig. 5).

\section{Sensitivity experiments}

A series of sensitivity experiments have been conducted. They deal with the dependency of the simulation to different initial states (Ji and Vernekar 1997; Rinke and Dethloff 2000; Weisse et al. 2000; Caya and Biner 2004) and with the role of higher resolution and with changed spectral nudging parameters. The dependency on different initial states is examined by considering a series of four different simulations that were initialized with analyses from different days at the beginning of August 1997. When spectral nudging is invoked the different nudged simulations differ very little amongst each other (Fig. 4), as was the case with northeast Atlantic climate in Weisse et al. (2000) and Weisse and Feser (2003). The large-scale constraint obviously steers the track of the cyclone tightly. This is quite different in the case of four simulations without spectral nudging. The tracks in three of four simulations deviate jointly quite a bit from the best track (Fig. 4), whereas one follows the best track much better.

When looking at the air pressure maps, one finds that Winnie is in all cases simulated, but that additional, unobserved typhoons were generated (Fig. 7). In one case, a strong tropical storm formed in the Bay of Bengal, while the other simulations just show a closed 1000$\mathrm{hPa}$ isobar. Satellite data or the best-track database as well as the NCEP reanalyses do not show a tropical storm in this area. This behavior is probably due to the fact that the process of forming tropical storms is a strongly nonlinear process involving chaotic dynamics. Thus slightly different initial conditions can lead to rather different developments in the interior of the simulation domain. Interestingly, Winnie formed in all four cases.

Kobayashi and Sugi (2004) showed that increased grid resolution improves the simulation of the Asian monsoon. Tropical cyclones occurred more frequently and with higher intensities with increasing model resolution in their global model. For their highest resolution of T213 they simulated about 6 times more tropical storms than with a resolution of T42, but still the number of simulated storms only reached about $73 \%$ of the observed number. Also a mesoscale model comparison (Nagata et al. 2001) showed that horizontal resolution enhancement from 50 to $10 \mathrm{~km}$ has a great impact on intensity prediction of a typhoon while the track prediction was unaffected by the increased grid resolution.

For this paper the CLM was run, with spectral nudging as described above, also with higher resolution of 18 

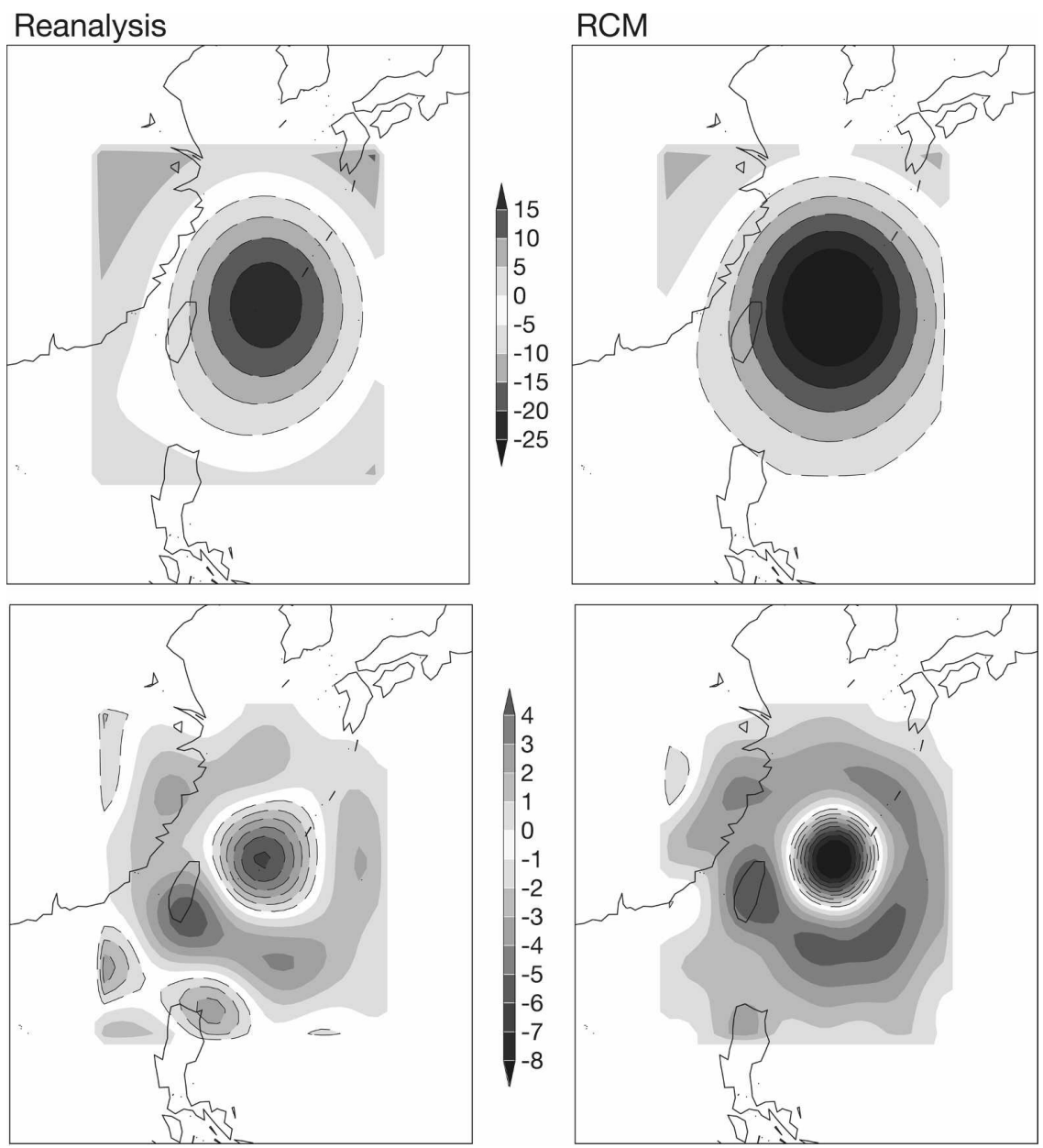

FIG. 6. Spatially filtered air pressure fields showing pressure deviation (hPa) at $0000 \mathrm{UTC}$ 17 Aug 1997. (left) NCEP-NCAR reanalysis after interpolation on a 50-km grid; (right) RCM simulation on a $50-\mathrm{km}$ grid. (top) Large scales retained (diameter $\geq 600 \mathrm{~km}$ ); (bottom) medium scales retained $(\geq 180 \mathrm{~km}$ and $\leq 360 \mathrm{~km})$. Negative values are also plotted as dashed isolines.

km using the "double nesting approach" (Fig. 1). The results of the $50-\mathrm{km}$ run were used as input for the higher-resolution run. The typhoon track in the highresolution simulation is very similar to the track in the $50-\mathrm{km}$ run (not shown). However, the core pressure is somewhat deeper, and the maximum near-surface winds slightly stronger (black dashed line in Fig. 5). The storm develops slower in the beginning, but intensifies quickly on 12 August, comparable to the development in the best-track data. Zhang et al. (2005) showed that Typhoon Winnie featured an outer and inner eyewall. They were able to reproduce this double eyewall with the fifth-generation Pennsylvania State UniversityNational Center for Atmospheric Research Mesoscale Model (MM5) at a resolution of $9 \mathrm{~km}$. In the current study only one eyewall could be simulated, probably because the finest resolution of $18 \mathrm{~km}$ was still too coarse to resolve these mesoscale structures.

In another experiment, the regional model was again run with a grid distance of $18 \mathrm{~km}$ for the small model domain of Fig. 1, but in this case the relatively coarse NCEP boundary conditions were directly taken as input for the $18-\mathrm{km}$ model. Interior nudging was used as well in this experiment. The CLM was nudged for wavenumbers matching the well-resolved scales of the NCEP reanalyses. This simulation yields clearly worse results than the $50-\mathrm{km}$ resolution run; the track becomes very noisy (not shown; it "spirals" around the main track). The core pressure and the maximum nearsurface winds become similar to the values in the driving NCEP reanalysis (black solid line in Fig. 5).

The dependency on the spatial scales used for the 
(a)

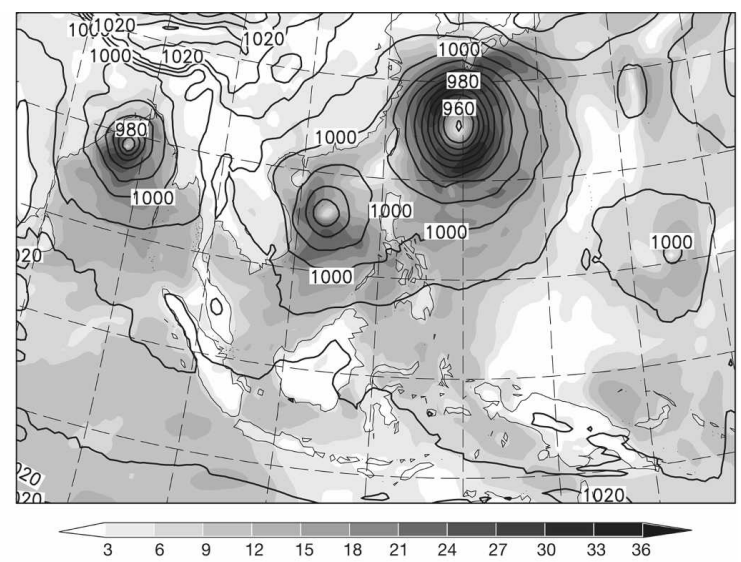

(b)

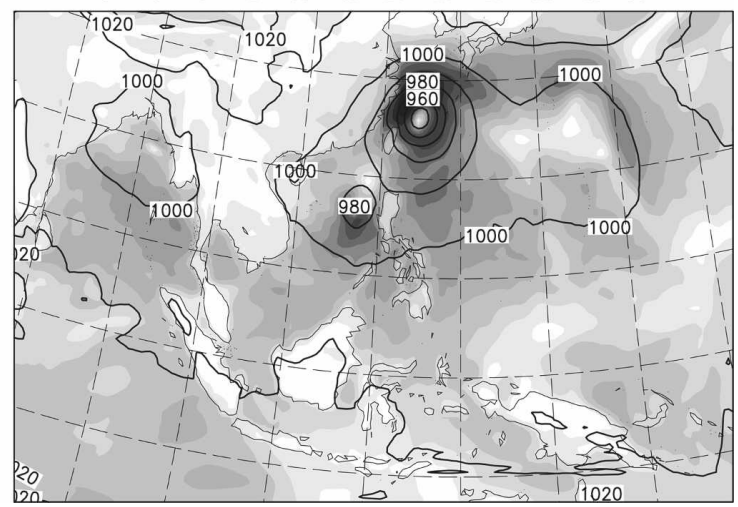

FIG. 7. Two simulations with CLM representing 0000 UTC 17 Aug 1997 on the 50-km grid without spectral nudging. The simulation was begun with (a) initial conditions of 0000 UTC 3 Aug 1997 and (b) initial conditions of 0000 UTC 7 Aug 1997. The figure shows sea level pressure isobars $(\mathrm{hPa})$ and shaded nearsurface wind speed fields $\left(\mathrm{m} \mathrm{s}^{-1}\right)$.

spectral nudging was also examined. Different simulations for both the CLM with $50-\mathrm{km}$ and with $18-\mathrm{km}$ grid distance were calculated. The spectral nudging was applied for scales larger than 2000 and $3000 \mathrm{~km}$ while the nudging coefficient was increased from 0.05 to 1.0. The results showed only small deviations from the runs with the spectral nudging configurations discussed before.

\section{Summary and conclusions}

In this work the authors wanted to assess the feasibility to transfer a high-resolution hindcast approach, which was successfully applied to western Europe (Feser et al. 2001), Southeast Asia, and the northwest Pacific. Thereby the main new task for the regional model is the simulation of typhoons. These ensemble simulations (with and without usage of spectral nudging) were computed in climate mode, so that any skill of reproducing the typhoon is not related to details of initial conditions. Case studies were computed to evalu- ate the model's ability in generating typhoons inside the model domain (without introducing an artificial vortex) and in improving core pressure and near-surface wind speed compared to the driving reanalyses. The tracks, core pressure, and wind speed developments of Typhoon Winnie were extracted from reanalyses and regional model runs and compared to best-track data. Sensitivity experiments were computed to test the effects of higher grid resolutions and spectral nudging configurations as well as the dependency of the simulations to their initial states. From this typhoon simulation study three conclusions can be drawn, which go beyond findings of previous studies:

1) Some typhoons can be described at least partly realistically with the dynamical downscaling concept, using coarse-grid NCEP reanalyses and regional atmospheric models-which is consistent with the success reported by Landman et al. (2005). In the case analyzed in this paper, Winnie from August 1997, the storm was captured and described by the reanalysis so that the RCM did not need to "invent" the storm. The tropical cyclone started to develop inside of the regional model domain and did not just pass through the lateral boundaries, at least not in any even minor developed stage. It is supposed that the initiation of the tropical cyclone development inside of the RCM domain is controlled by the largescale forcing data, both for the $0.5^{\circ}$ simulation forced by reanalyses as well as for the $0.165^{\circ}$ simulation forced by the $0.5^{\circ} \mathrm{CLM}$ run. The storm was simulated in greater detail than in the NCEP description; also the core pressure was considerably deeper and the winds stronger. Further experiments need to be made with less strong storms; first results indicate that sometimes such less energetic storms are not captured as well.

2) The presented experiments indicate that lateral boundary control is insufficient to reconstruct the number and track of typhoons in a given synoptic situation. Of course, this depends on the size of the domain; in smaller domains the lateral control is more efficient (Castro et al. 2005). The inclusion of large-scale constraints, as for instance spectral nudging, is helpful in forming the storms along the right track.

3) Using a double-nesting approach has an effect on the simulation, which is believed to be a slight improvement compared to the $50-\mathrm{km}$ simulation in terms of core pressure development and nearsurface wind speed. But the typhoon tracks were very similar; this finding is in compliance with the results of Nagata et al. (2001). 
It is stressed that the reported results concern just one case. Before engaging in a multidecadal retrospective simulation more cases and specific storm-rich and storm-poor seasons will be examined. Thereby it will assessed whether they return the right storm statistics (in frequency, location, and intensity) conditional upon a number of large-scale factors, such as SST, vertical stability, and the like.

A major problem for this analysis is the lack of a reliable database for typhoons in Southeast Asia that homogeneously describes air pressure fields, wind speed fields, and other meteorological variables to evaluate the regional climate model simulations in more detail. Only recently, Zhang et al. (2007) presented a high-resolution tropical cyclone reanalysis for selected western Pacific typhoons of the year 2004, which has great potential for regional model validation efforts.

The authors think that the results of their case study are promising so that the next step will be to compare different typhoon seasons with many and few typhoons. Afterward a multidecadal hindcast for Southeast Asia will be computed resembling the approach presented by Knutson et al. (2007) for Atlantic hurricanes. The regional atmospheric model data will serve as input for wave, sea level, and storm surge models. These results should enable the assessment of possible changes in the atmospheric and maritime climate for Southeast Asia during the last decades.

Acknowledgments. The authors thank B. Rockel for valuable discussions regarding this work. B. Gardeike helped with the preparation of the figures for this paper. Three anonymous reviewers gave constructive comments that helped to improve the paper. The climate version of the "Lokal-Modell" is the community model of the German climate research. The authors also thank the German Climate Computing Center (DKRZ), which provided the computer hardware for the LAM simulations in the project "Reconstructions and Scenarios." The NCEP-NCAR reanalyses data were provided by the National Center for Atmospheric Research (NCAR).

\section{REFERENCES}

Aspelien, T., 2006: The use of long-term observations in combination with modeling and their effect on the estimation of the North Sea storm surge climate. Ph.D. thesis, University of Hamburg, 106 pp.

Bärring, L., and H. von Storch, 2004: Scandinavian storminess since about 1800. Geophys. Res. Lett., 31, L20202, doi:10.1029/2004GL020441.

Camargo, S., H. Li, and L. Sun, 2007: Feasibility study for down- scaling seasonal tropical cyclone activity using the NCEP regional spectral model. Int. J. Climatol., 27, 311-325.

Castro, C. L., R. A. Pielke Sr., and G. Leoncini, 2005: Dynamical downscaling: Assessment of value retained and added using the Regional Atmospheric Modeling System (RAMS). J. Geophys. Res., 110, D05108, doi:10.1029/2004JD004721.

Caya, D., and S. Biner, 2004: Internal variability of RCM simulations over an annual cycle. Climate Dyn., 22, 33-46.

Chan, J. C. L., Y. M. Liu, K. C. Chow, Y. H. Ding, W. K. M. Lau, and K. L. Chan, 2004: Design of a regional climate model for the simulation of South China summer monsoon rainfall. $J$. Meteor. Soc. Japan, 82, 1645-1665.

Davies, H. C., 1976: A lateral boundary formulation for multilevel prediction models. Quart. J. Roy. Meteor. Soc., 102, 405418.

Feser, F., 2006: Enhanced detectability of added value in limitedarea model results separated into different spatial scales. Mon. Wea. Rev., 134, 2180-2190.

—_, and H. von Storch, 2005: A spatial two-dimensional discrete filter for limited-area-model evaluation purposes. Mon. Wea. Rev., 133, 1774-1786.

- R. Reisse, and H. von Storch, 2001: Multi-decadal atmospheric modeling for Europe yields multi-purpose data. Eos, Trans. Amer. Geophys. Union, 82, 305-310.

Fu, C., and Coauthors, 2005: Regional Climate Model Intercomparison Project for Asia. Bull. Amer. Meteor. Soc., 86, 257266.

Gaslikova, L., and R. Weisse, 2006: Estimating near-shore wave statistics from regional hindcasts using downscaling techniques. Ocean Dyn., 56, 26-35.

Hodges, K. I., 1994: A general method for tracking analysis and its application to meteorological data. Mon. Wea. Rev., 122, 2573-2586.

, 1995: Feature tracking on the unit sphere. Mon. Wea. Rev., 123, 3458-3465.

Ji, Y., and A. D. Vernekar, 1997: Simulation of the Asian summer monsoons of 1987 and 1988 with a regional model nested in a global GCM. J. Climate, 10, 1965-1979.

Kain, J. S., 2004: The Kain-Fritsch convective parameterization: An update. J. Appl. Meteor., 43, 170-181.

Kalnay, E., and Coauthors, 1996: The NCEP/NCAR 40-Year Reanalysis Project. Bull. Amer. Meteor. Soc., 77, 437-471.

Kanamaru, H., and M. Kanamitsu, 2007: Scale-selective bias correction in a downscaling of global analysis using a regional model. Mon. Wea. Rev., 135, 334-350.

Kang, H.-S., D.-H. Cha, and D.-K. Lee, 2005: Evaluation of the mesoscale model/land surface model (MM5/LSM) coupled model for East Asian summer monsoon simulations. J. Geophys. Res., 110, D10105, doi:10.1029/2004JD005266.

Kida, H., T. Koide, H. Sasaki, and M. Chiba, 1991: A new approach for coupling a limited area model to a GCM for regional climate simulations. J. Meteor. Soc. Japan, 69, 723-728.

Knutson, T. R., J. J. Sirutis, S. T. Garner, I. M. Held, and R. E. Tuleya, 2007: Simulation of the recent multidecadal increase of Atlantic hurricane activity using an $18-\mathrm{km}$-grid regional model. Bull. Amer. Meteor. Soc., 88, 1549-1565.

Kobayashi, C., and M. Sugi, 2004: Impact of horizontal resolution on the simulation of the Asian summer monsoon and tropical cyclones in the JMA global model. Climate Dyn., 93, 165-176.

Landman, W. A., A. Seth, and S. J. Camargo, 2005: The effect of regional climate model domain choice on the simulation of tropical cyclone-like vortices in the southwestern Indian Ocean. J. Climate, 18, 1263-1274. 
Laprise, R., 2003: Resolved scales and nonlinear interactions in limited-area models. J. Atmos. Sci., 60, 768-779.

Lee, D.-K., D.-H. Cha, and H.-S. Kang, 2004: Regional climate simulation of the 1998 summer flood over East Asia. J. Meteor. Soc. Japan, 82, 1735-1753.

Liu, Y. M., J. C. L. Chan, K. C. Chow, and Y. H. Ding, 2006: Ten-year climatology of summer monsoon over South China and its surroundings simulated from a regional climate model. Int. J. Climatol., 26, 141-157.

Miguez-Macho, G., G. L. Stenchikov, and A. Robock, 2004: Spectral nudging to eliminate the effects of domain position and geometry in regional climate model simulations. J. Geophys. Res., 109, D13104, doi:10.1029/2003JD004495.

Nagata, M., and Coauthors, 2001: A mesoscale model intercomparison: A case of explosive development of a tropical cyclone (COMPARE III). J. Meteor. Soc. Japan, 79, 999-1033.

Nakazawa, T., 2001: Suppressed tropical cyclone formation over the western North Pacific in 1998. J. Meteor. Soc. Japan, 79, 173-183.

Rinke, A., and K. Dethloff, 2000: On the sensitivity of a regional Arctic climate model to initial and boundary conditions. Climate Res., 14, 101-113.

Soares, C. G., R. Weisse, J. C. Carretero, and E. Alvarez, 2002: A 40 years hindcast of wind, sea level and waves in European waters. Proc. OMAE 2002: 21st Int. Conf. on Offshore Mechanics and Arctic Engineering, Oslo, Norway, American Society of Mechanical Engineers, 28604.

Sotillo, M. G., A. W. Ratsimandresy, J. C. Carretero, A. Bentamy, F. Valero, and F. González-Rouco, 2005: A high-resolution 44-year atmospheric hindcast for the Mediterranean Basin: Contribution to the regional improvement of global reanalysis. Climate Dyn., 25, 219-236.

Steppeler, J., G. Doms, U. Schättler, H. W. Bitzer, A. Gassmann, U. Damrath, and G. Gregoric, 2003: Meso-gamma scale forecasts using the nonhydrostatic model LM. Meteor. Atmos. Phys., 82, 75-96.

von Storch, H., 1995: Inconsistencies at the interface of climate impact studies and global climate research. Meteor. Z., 4, $72-80$.

, H. Langenberg, and F. Feser, 2000: A spectral nudging technique for dynamical downscaling purposes. Mon. Wea. Rev., 128, 3664-3673.

Waldron, K. M., J. Paegle, and J. D. Horel, 1996: Sensitivity of a spectrally filtered and nudged limited-area model to outer model options. Mon. Wea. Rev., 124, 529-547.

Walsh, K. J. E., K.-C. Nguyen, and J. L. McGregor, 2004: Fineresolution regional climate model simulations of the impact of climate change on tropical cyclones near Australia. Climate Dyn., 22, 47-56.

Weisse, R., and F. Feser, 2003: Evaluation of a method to reduce uncertainty in wind hindcasts performed with regional atmosphere models. Coastal Eng., 48, 211-225.

_ , and A. Plüss, 2006: Storm-related sea level variations along the North Sea coast as simulated by a high-resolution model 1958-2002. Ocean Dyn., 56, 16-25.

- - H. Heyen, and H. von Storch, 2000: Sensitivity of a regional atmospheric model to a sea state-dependent roughness and the need of ensemble calculations. Mon. Wea. Rev., 128, 3631-3642.

- H. von Storch, and F. Feser, 2005: Northeast Atlantic and North Sea storminess as simulated by a regional climate model during 1958-2001 and comparison with observations. J. Climate, 18, 465-479.

Wright, D. G., K. R. Thompson, and Y. Lu, 2006: Assimilating long-term hydrographic information into an eddy-permitting model of the North Atlantic. J. Geophys. Res., 111, C09022, doi:10.1029/2005JC003200.

Zhang, Q.-H., S.-J. Chen, Y.-H. Kuo, K.-H. Lau, and R. A. Anthes, 2005: Numerical study of a typhoon with a large eye: Model simulation and verification. Mon. Wea. Rev., 133, 725742.

Zhang, X., T. Li, F. Weng, C.-C. Wu, and L. Xu, 2007: Reanalysis of western Pacific typhoons in 2004 with multi-satellite observations. Meteor. Atmos. Phys., 97, 3-18. 